\title{
Performance Evaluation of Network Mobility Handover over Future Aeronautical Data Link
}

\author{
Serkan Ayaz ${ }^{*, a, b}$, Felix Hoffmannª, Ulrich Epple ${ }^{\mathrm{a}}$, Reinhard German ${ }^{\mathrm{b}}$, Falko Dressler ${ }^{\mathrm{c}}$ \\ ${ }^{a}$ German Aerospace Center (DLR), Institute of Communications and Navigation, Wessling, Germany \\ ${ }^{b}$ Computer Networks and Communication Systems, Dept. of Computer Science, University of Erlangen, Germany \\ ${ }^{c}$ Computer and Communication Systems, Institute of Computer Science, University of Innsbruck, Austria
}

\begin{abstract}
The aviation community is currently working on the standardization of data communication systems for the future air traffic management. In this context, International Civil Aviation Organization (ICAO) has initiated a work on standardization of an IPv6-based aeronautical telecommunications network and on future radio access technologies, respectively. In this paper, we integrate L-Band Digital Aeronautical Communications System Option 1 (L-DACS 1), which is one candidate for future radio access technologies, with realistic IPv6-based network layer functionality and analyze the effect of handover delay to the TCP performance. Realistic Frame Error Rate (FER) values obtained from an L-DACS 1 physical layer simulator, which uses a realistic aeronautical channel model, are used in the simulation experiments. In the first stage, we decreased layer 3 handover latency by removing the Duplicate Address Detection (DAD) procedure for address configuration. In the second stage, we introduced a Home Agent (HA)-buffering method, which is used to buffer the traffic (destined to the mobile node) during handover. Transmission completion time is the primary performance metric in our analysis. With the HA-buffering method, the transmission completion time is reduced by at least $10 \%$ for the transmission of $110 \mathrm{kB}$ of information over a wireless link with $31.5 \mathrm{kbit} \mathrm{s}^{-1}$ data rate.
\end{abstract}

Key words: Aviation, Mobile IPv6, Network Mobility, Handover, Home Agent Buffering, TCP

\section{Introduction}

In Air Traffic Management (ATM), there are two main communication services: Air Traffic Services (ATS) and Airline Operations Services (AOS)[1]. ATS Correspondent Nodes (CNs) are used to provide navigation, control, and situational awareness services to the aircraft, whereas AOS CNs are mainly used for business operations of airline companies. Using today's communication technologies, these services are generally performed by using analogue voice communications. However, digital data communication utilizes the bandwidth more efficiently and overall is much less error-prone than analogue voice communication. In addition, only with the digital data communications new ATM concepts like 4D trajectory exchange and graphical weather information transmission are possible.

For this reason, two main activities are running in parallel in order to build a future aeronautical communications network. ICAO is working on the one side on the standardization of the next generation IPv6-based Aeronautical Telecommunications Network (ATN/IPS)[2] and, on the other side, on the standardization of future radio access technologies for aeronautics.

In this work, we analyze the effect of handover performance of the L-DACS 1 integrated with the IPv6 network layer func-

\footnotetext{
${ }^{*}$ Corresponding author.

Email addresses: serkan. ayaz@dlr.de (Serkan Ayaz), felix.hoffmann@dlr.de (Felix Hoffmann), ulrich.epple@dlr.de (Ulrich Epple), german@inf ormatik . uni-erlangen.de (Reinhard German), dressler@ieee.org (Falko Dressler)
}

tionality including Network Mobility (NEMO) support[3] on the TCP performance.

\subsection{Related Work}

Mobile IPv6 (MIPv6) and its extensions are very well studied protocols by the research community. Previous studies mainly considered link technologies from the domain of consumer electronics. For example, a simulation study of MIPv6 on IEEE $802.11 \mathrm{~b}$ provides a performance evaluation of different smart handover extensions including link layer triggers for MIPv6[4]. Another work presents testbed experiments related to the use of MIPv6 with IEEE 802.11g technology[5]. Similar results have been presented in[6]. Here, MIPv6 has been studied in an experimental testbed using WiMAX and $\mathrm{WiFi}$ as underlying link layer technologies. In[7], NEMO is used as a base protocol in a testbed where two different 802.11 interface cards are used in order to perform make-before-break handovers.

In the aeronautical domain another set of link technologies is considered, which mainly differ due to their data rate and cell size. Currently deployed link technologies provide data rates in the range of 3-30 $\mathrm{kbit} \mathrm{s}^{-1}$ per cell. However, future radio access technologies like L-DACS 1 , which this paper focuses on, provide data rates in the range of $291-1318 \mathrm{kbit} \mathrm{s}^{-1}$ in the Forward Link (FL) and 270-1267 $\mathrm{kbit} \mathrm{s}^{-1}$ in the Return Link (RL) per cell depending on selected modulation and coding scheme. Although L-DACS 1 increases the data rate beyond that provided by current aeronautical links, the link capacity is still far behind that of consumer electronics. Another difference is 


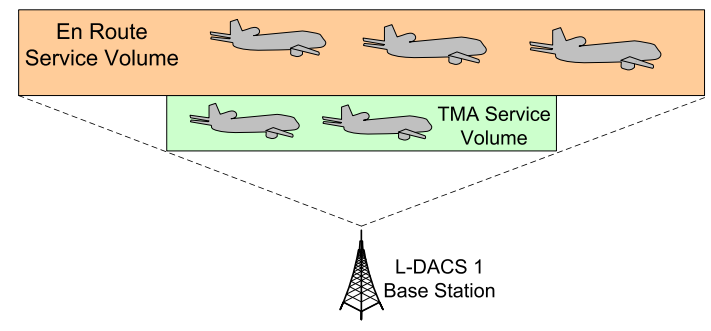

Figure 1: Airspace organization

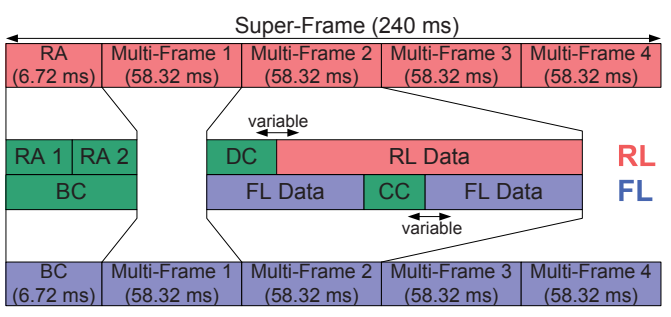

Figure 2: L-DACS 1 frame structure the cell size and the number of mobile users. Typical cell radius are in the range of $150-250 \mathrm{~km}$ and if we assume one Terminal Maneuvering Area (TMA) and En Route service volume[8] is covered by one cell (cf. Fig. 1), then each cell is providing services for up to around 90 aircraft (abbreviated as " $\mathrm{a} / \mathrm{c}$ " in the rest of this paper).

\subsection{Contributions}

In our previous studies, we presented an initial step towards investigations of MIPv6 / NEMO handover delay in an aeronautical environment $[9,10]$. In[9], we only considered generic link layers with certain bandwidth and delay values, whereas in[10], we also studied the impact of a realistic link layer (L-DACS 1) including an Automatic Repeat Request (ARQ) component. We provided different proposals in order to decrease the signaling overhead and better handover delay performance. In this study, we extend our previous work in[10] as follows:

- new proposals to reduce the handover delay due to the DAD procedure for Home Address (HoA) and Care-of Address (CoA) selection are discussed,

- more realistic FER values have been achieved from the L-DACS 1 physical layer simulator[11],

- a generic TCP analysis and parameter tuning, i.e. Maximum Segment Size (MSS) and receiver window size (rwnd), provided in order to use it effectively with L-DACS 1, and

- a HA-buffering method is presented in order to improve TCP performance during inter-access network handovers.

In the scope of this paper, we consider single interface interaccess network handovers with the NEMO protocol as a base mobility management protocol.

\section{L-Band Digital Aeronautical Communications System Option 1}

Within ICAO currently two candidate radio access technologies for the future provision of ATS and AOS services in the L-band are considered ${ }^{1}$. These technologies are referred to as L-DACS 1 and L-DACS 2. Initial specifications for both technologies have been published by EUROCONTROL[12, 13], and

\footnotetext{
${ }^{1}$ see http: //www. eurocontrol. int/communications/.
}

it is planned that one of these two systems will become operational around 2020.

L-DACS 1 has been designed for the transmission of both digital voice and data. In L-DACS 1, RL and FL are separated by means of Frequency Division Duplex (FDD). In the RL, a combination of Orthogonal Frequency Division Multiple Access (OFDMA) and Time Division Multiple Access (TDMA) is used, whereas in the FL, Orthogonal Frequency Division Multiplexing (OFDM) is applied. The TDMA component in the RL is selected in order to minimize the possibility of interference with legacy systems which are operating in the L-band (e.g. distance measuring equipment (DME)). This is important since an L-DACS 1 transmitter operates close to other receivers on board, so it should only be active for a short time, reducing these receivers' exposure to interference.

\subsection{Frame Structure and Resource Allocation}

The frame structure is shown in Fig. 2. Time is divided into superframes with a duration of $240 \mathrm{~ms}$. At the beginning of each superframe, a/c have the opportunity to log onto the network using a Random Access Channel (RACH), whereas the Base Station (BS) transmits general cell information in the Broadcast Channel (BCCH). The rest of the superframe consists of four multiframes, each with a duration of $58.32 \mathrm{~ms}$ and consisting of both data and control frames. In the FL, the BS transmits control information, such as resource allocation, i.e. FL mapping and RL mapping, and acknowledgments on the Common Control Channel $(\mathrm{CCCH})$. In the $\mathrm{RL}$, each $\mathrm{a} / \mathrm{c}$ is assigned one slot per multiframe within the Dedicated Control Channel (DCCH) for the transmission of control data. At most, $52 \mathrm{a} / \mathrm{c}$ can be accommodated within the DCCH. If more $\mathrm{a} / \mathrm{c}$ are registered with a single $\mathrm{BS}$, a/c will not receive a $\mathrm{DCCH}$ slot in every multiframe. Both the $\mathrm{CCCH}$ and $\mathrm{DCCH}$ are of variable length to allow efficient use of the wireless resources.

Before any transmission can take place, either in the RL or the FL, resources must be requested from the BS. At the beginning of a $\mathrm{CCCH}$ slot, the $\mathrm{BS}$ considers all received resource requests (sent via an RSRC_RQST message) since the last $\mathrm{CCCH}$ slot. It allocates resources, i.e. TDMA slots and OFDMA subchannels, for the $\mathrm{a} / \mathrm{c}$, and informs the $\mathrm{a} / \mathrm{c}$ via an RSRC_RESP message. The exact scheduling algorithm to be used by the BS is left open by the L-DACS 1 specification. In our implementation, we have adopted static priority queuing, i.e. requests with the highest priority are fulfilled first and each priority class uses deficit round robin with fragmentation scheduling mechanism[14]. The allocation of resources is broadcast to all a/c in the $\mathrm{CCCH}$ slot, 


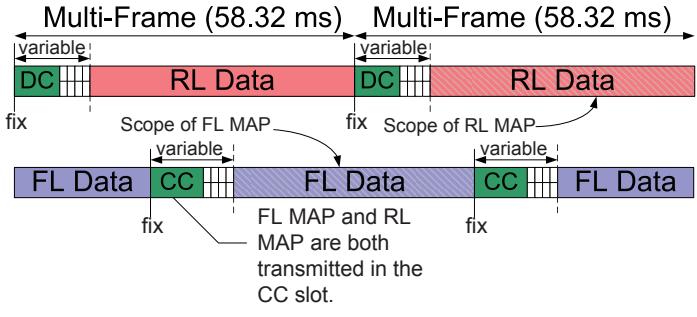

Figure 3: L-DACS 1 resource allocation structure

specifying which $\mathrm{a} / \mathrm{c}$ is allowed to transmit when and on which subchannels. The scope of this resource allocation is shown in Fig. 3.

\subsection{Automatic Repeat Request Mechanism}

L-DACS 1 supports both unacknowledged and acknowledged data transfer modes. Due to the rigid frame structure of the L-DACS 1 protocol, a transmitter knows when it should expect an acknowledgment for data that it has transmitted. After one missed acknowledgment opportunity, a packet is retransmitted. After a certain number of subsequent retransmissions, the entire transmission is aborted and the packet is discarded at the transmitter. Note that the entire process of resource request and allocation must again be performed before the lost packet can be retransmitted.

\subsection{Handover Types}

Two different types of handover are foreseen by the L-DACS 1 specification. In both handover types, the BS polls the a/c to provide power reports of their received signal strength. Polling of neighboring cells' received signal strength is requested by transmitting the Adjacent Cell Broadcast (ACB) and Scanning Table Broadcast (STB) messages in the BCCH channel. In the upcoming $\mathrm{BCCH}$ channel, the a/c switches to the next BS frequency and measures the received power by listening to the System Identification Broadcast (SIB) message from this BS. It then sends a POW_REP power report message to the current BS. If the adjacent cell's received power level is higher than that of the current cell, the current BS triggers a но_сом handover message to this cell.

In the case of a type 1 handover, the a/c simply confirms the handover, sends a CELL_EXIT message to the current BS, and switches to the channel of the next BS, where it registers via the new station's RACH by sending a CELL_RQST message. If no collision has occurred on the $\mathrm{RACH}$, the BS will respond with a CELL_RESP on the CCCH and assign a subscriber access code and a DCCH slot to the a/c. In the case of a collision on the $\mathrm{RACH}$, the a/c does not receive this response and will perform an exponential backoff, attempting to access the RACH again later.

In this paper, we only consider type 1 handover since it does not require any signaling between BSs and is more suitable for inter-access network handovers. The details of type 2 handover can be found in L-DACS 1 specification[12].

\subsection{Received Power and Wireless Channel Errors}

In our simulation experiments, we consider a free-space path loss model for the wireless channel since we mainly focus on the En Route service volume in which the line-of-sight (LOS) component is very strong [15]. The received power $P_{r x}$ is the main parameter that is used for the handover process as defined in Eqn. 1. It depends on the transmit power $P_{t x}$, the receiver and transmitter antenna gains $G_{r x}$ and $G_{t x}$, cable and diplexer losses in the transmitter as well as in the receiver (i.e. $L_{\mathrm{tx}}, L_{\mathrm{rx}}$ ), the transmit center frequency $f_{t x}$, and on the distance $d$ between transmitter and receiver. The particular values are summarized in Table 1 for both, FL and RL transmission, adopted from the L-DACS 1 link budget calculation in [12].

Given these values, the received power is obtained by

$$
P_{r x}=P_{\mathrm{tx}}+G_{\mathrm{rx}}+G_{\mathrm{rx}}-L_{\mathrm{tx}}-L_{\mathrm{rx}}-20 \log \left(\frac{c}{4 \cdot \pi \cdot d \cdot f_{\mathrm{tx}}}\right)(1)
$$

assuming $c=3 \cdot 10^{8} \mathrm{~m} / \mathrm{s}$; power, gain, and loss values given in $\mathrm{dB}$.

In addition, in order to reflect the user data losses in the wireless channel during the handover (i.e., when the $\mathrm{a} / \mathrm{c}$ is close to the boundary of the cell), we consider realistic FER values that are obtained from L-DACS 1 physical layer simulator [11]. The simulations take a realistic En route channel model as well as interference, induced by the DME, which operates in the same frequency range, into account. The interference scenario and the applied interference mitigation technique were adopted from [16]. For estimating the transmission channel, a pilot based linear interpolation was implemented. In Fig. 4, FER and Bit Error Rate (BER) curves are plotted versus the Signal-to-Noise Ratio (SNR). Regarding coding and modulation, L-DACS 1 supports adaptive coding and modulation, which are chosen according to the channel conditions, receive power and maximizing the throughput. In our case, we choose the most robust setup of Quadrature Phase Shift Keying (QPSK) and a concatenation of a rate $1 / 2$ convolutional code and a rate 0.9 Reed-Solomon code since $\mathrm{a} / \mathrm{c}$ is flying near to the cell boundary. For an L-DACS 1 transmission, a BER of $1 \cdot 10^{-6}$ in the FL data frames is required according to[12]. As depicted in Fig. 4, this rate is achieved at $\mathrm{SNR}=8.7 \mathrm{~dB}$.

This leads to FER values of $6.28 \cdot 10^{-2}$ for the RL and 6.31 . $10^{-5}$ for the FL data slots at the considered SNR of $8.7 \mathrm{~dB}$ as shown in Fig. 4. Each FL and RL data frame carries 91 byte and 14 byte, respectively. Note that, these FER values are the main input values for the radio channel module in our simulations in section 5. We also assume all L-DACS 1 specific messages (i.e. control frames) are transmitted without any channel error in our simulations.

Link budget calculations, given in Table 2, indicate that for a typical cell size of $225 \mathrm{~km}$ and exemplary chosen transmit frequencies of $f_{\mathrm{tx}, \mathrm{FL}}=993.5 \mathrm{MHz}$ in the FL and $f_{\mathrm{tx}, \mathrm{RL}}=$ 1056.5 MHz in the RL, the SNR working point of 8.7 dB leads to a positive link budget, indicated by a positive system operating margin, $O_{\mathrm{m}}$. In the table, thermal noise density $\left(N_{0}\right)$ is calculated as $10 \log (k T)$ where $k=1.381 \cdot 10^{-23} \mathrm{~J} / K$ (Boltzmann constant) and $T=290 K$ (Temperature). Note that compared to the link 


\begin{tabular}{lll}
\hline Parameter & FL & RL \\
\hline Tx power, $P_{\mathrm{tx}}$ & $41 \mathrm{dBm}$ & $41 \mathrm{dBm}$ \\
Tx antenna gain, $G_{\mathrm{tx}}$ & $8 \mathrm{dBi}$ & $0 \mathrm{dBi}$ \\
Tx cable \& diplexer loss, $L_{\mathrm{tx}}$ & $2 \mathrm{~dB}$ & $3.5 \mathrm{~dB}$ \\
Tx center frequency, $f_{\mathrm{tx}}$ & {$[985.5-1008.5] \mathrm{MHz}$} & {$[1048.5-1071.5] \mathrm{MHz}$} \\
Tx-Rx distance, $d$ & $225 \mathrm{~km}$ & $225 \mathrm{~km}$ \\
Rx antenna gain, $G_{\mathrm{rx}}$ & $0 \mathrm{dBi}$ & $8 \mathrm{dBi}$ \\
Rx cable \& diplexer loss, $L_{\mathrm{rx}}$ & $3.5 \mathrm{~dB}$ & $2 \mathrm{~dB}$ \\
\hline
\end{tabular}

Table 1: Values of L-DACS 1 transmission

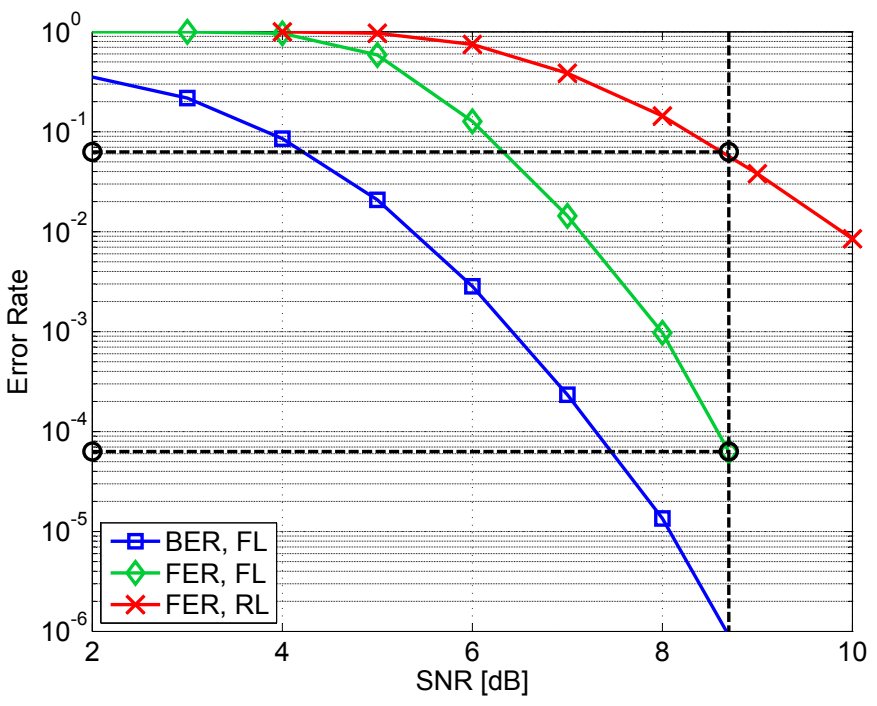

Figure 4: Error rates versus SNR for L-DACS 1 transmission

budget in [12], no implementation margin was considered, since we applied a realistic channel estimation in our physical layer simulations.

\section{Network Mobility}

Network Mobility (NEMO)[3] introduces the concept of Mobile Routers (MRs), which extends the mobile host functionality[17] in such a way that it provides IP addresses to the connected Mobile Network Nodes (MNNs) from the assigned Mobile Network Prefix (MNP). The assigned MNP is associated with the HoA of the MR.

\subsection{Basic NEMO Operation}

When a $\mathrm{CN}$ sends a packet to a MNN of the MR, the packet is first routed to the HA that advertises MNP (MNNs are configuring their IP addresses from the MNP). After the HA receives the packet, it checks its Binding Cache Entry $(\mathrm{BCE})^{2}$ in order to tunnel the packet to the current point of network attachment of MR (i.e., to the CoA of the MR). On the reverse path (i.e., when a $\mathrm{MNN}$ sends a packet to its $\mathrm{CN}$ ), the MR receives the packet

\footnotetext{
${ }^{2}$ Each BCE mainly contains HoA, CoA and MNPs of the mobile node with a lifetime value indicating the remaining lifetime for this entry.
}

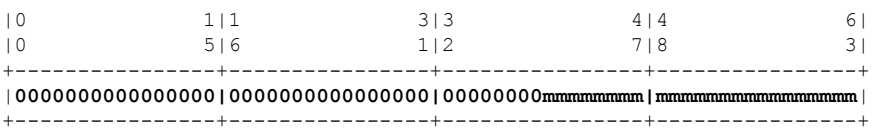

Figure 5: Creating modified EUI-64 format interface identifiers

from the MNN and tunnels it to the HA. When the HA receives the packet, it decapsulates the packet and finally sends it to the $\mathrm{CN}$.

\subsection{Providing Unique IPv6 Addresses}

As described in[10], DAD time for HoA and CoA selection during the handover increases the handover completion time. Two different approaches are considered in order to guarantee the uniqueness of the generated HoA and CoA, so that the DAD procedure can be removed from the handover process.

Reference [2] provides /32 IPv6 address prefix format for the mobile nodes[2]. However, this does not provide information on how an interface identifier, which corresponds to the least significant 64 bits, can be configured.

In the aeronautical domain, each a/c has a unique 24-bit ICAO address. We propose to use this address for the configuration of the a/c's interface identifier field for the CoA. As defined in Appendix A of[18], a modified 64-bit extended unique identifier (EUI-64) format can be created by taking the 24-bit ICAO address prefixed with binary zero as shown in Fig. 5. In addition, a unique Home Network Prefix (HNP) can be assigned to each MR during the initial network connection phase (the bootstrapping phase) similar to[19] or via IKEv2 as specified in[20], so that the generated HoA from unique HNP is implicitly unique.

Using these two adaptations, the total handover latency is reduced to the home registration and layer 2 handover procedures.

\section{The Proposed Handover Scheme for Inter Access Net- work Handovers}

In order to optimize the TCP performance during handovers, there are four important approaches, which are related to MIPv6/ NEMO protocols. Table 3 summarizes these approaches, namely HA-buffering[21, 22], HA bi-casting[23], BS-buffering[24, 25] and Access Router (AR)-buffering[26, 27] . NB: there are also cross layer approaches, which are not shown in Table 3. These approaches require certain modifications either on the TCP sender and/or the receiver side[28, 29, 30]. 


\begin{tabular}{llll}
\hline Parameter & FL & RL & Formula \\
\hline Rx power, $P_{\mathrm{rx}}$ & $-95.26 \mathrm{dBm}$ & $-95.80 \mathrm{dBm}$ & see Eqn. 1 \\
Safety margin, $M_{\mathrm{s}}$ & $6 \mathrm{~dB}$ & $6 \mathrm{~dB}$ & \\
Thermal noise density, $N_{0}$ & $-174 \mathrm{dBm} / \mathrm{Hz}$ & $-174 \mathrm{dBm} / \mathrm{Hz}$ & \\
Tx bandwidth, $B$ & $498.05 \mathrm{kHz}$ & $498.05 \mathrm{kHz}$ & \\
Thermal noise power, $P_{\mathrm{N} 0}$ & $-117.03 \mathrm{dBm}$ & $-117.03 \mathrm{dBm}$ & $N_{0}+10 \log (B)$ \\
Rx noise figure, NF & $6 \mathrm{dBm}$ & $5 \mathrm{dBm}$ & \\
Rx noise power, $P_{\mathrm{N}, \mathrm{rx}}$ & $-111.03 \mathrm{dBm}$ & $-112.03 \mathrm{dBm}$ & $P_{\mathrm{N} 0}+\mathrm{NF}$ \\
Target SNR & $8.7 \mathrm{~dB}$ & $8.7 \mathrm{~dB}$ & \\
Rx sensitivity, $S_{0}$ & $-102.33 \mathrm{~dB}$ & $-103.33 \mathrm{~dB}$ & $P_{\mathrm{N}, \mathrm{rx}}+\mathrm{SNR}$ \\
Rx operation point, $S_{1}$ & $-96.33 \mathrm{~dB}$ & $-97.33 \mathrm{~dB}$ & $S_{0}+M_{\mathrm{s}}$ \\
System operating marging, $O_{\mathrm{m}}$ & $1.07 \mathrm{~dB}$ & $1.53 \mathrm{~dB}$ & $P_{\mathrm{rx}}-S_{1}$ \\
\hline
\end{tabular}

Table 2: Link budget for L-DACS 1

\begin{tabular}{lcccc}
\hline & $\begin{array}{c}\text { HA-buffering } \\
{[21,22]}\end{array}$ & $\begin{array}{c}\text { HA-bicasting } \\
{[23]}\end{array}$ & $\begin{array}{c}\text { BS-buffering } \\
{[24,25]}\end{array}$ & $\begin{array}{c}\text { AR-buffering } \\
{[26,27]}\end{array}$ \\
\hline Inter-AN Handover & $\checkmark$ & $\checkmark$ & $x$ & $x$ \\
Intra-AN Handover & $\checkmark$ & $\checkmark$ & $\checkmark$ & $\checkmark$ \\
\hline
\end{tabular}

Table 3: Layer 3 TCP optimizations during handover

BS-buffering and AR-buffering methods are only suitable for intra-access network handovers, since they require signaling between access routers or base stations and such kind of collaboration and trust relationship may not be possible between different access network service providers. In addition, these approaches require major modifications on the existing access network infrastructure (i.e., software update and configuration of all BSs and ARs). Furthermore, these protocols only support host mobility (Mobile IPv4 (MIPv4) or MIPv6) and not NEMO. Another proposal, HA bi-casting, works for intra and inter-access network handovers. However, it has three main drawbacks. One is the static configuration of a mobility database on the mobile router, which stores the list of access routers, their network prefixes, and possible CoAs for each candidate access network. Furthermore, the bi-casting of the traffic causes two times more traffic load on the ground network. Finally, since the bi-casted packets are not stored in the network during the handover, packet losses are inevitable during the handover. In this paper, we focus on HA-buffering, which is not only suitable for single-interface inter access network handovers but also intra-access network handovers. It does not require any static configuration on the a/c as opposed to HA bi-casting and it does not require any major modification on the access network as opposed to BS-buffering and AR-buffering methods.

Our contribution to the HA-buffering protocol proposed in[21] is the analysis of its applicability to the ATN/IPS and is the integration with L-DACS 1 data link. We modified the L-DACS 1 handover mechanism and show its applicability with HA-buffering through simulations. To the best of our knowledge, there is not any simulation or implementation studies performed until now for HA-buffering method.

Fig. 6 depicts the scenario where an a/c is communicating with the $\mathrm{CN}$ over BS1. The $\mathrm{a} / \mathrm{c}$ is sending the received power level of the FL $\left(P_{B S 1}\right)$ to BS1 with POW_REP message in the DCCH channel. Whenever $P_{B S 1}$ drops below a certain threshold $T h_{1}$, BS1 requests the scanning of the neighboring BSs with $\mathrm{ACB}$ and STB messages transmitted in the $\mathrm{BCCH}$ channel. The a/c reports the received power levels back to BS1. This procedure continues until the received power level $P_{B S 2}$ of a certain BS (in our example BS2) is better compared to the current one. Thus, when $P_{B S 2}$ is higher than $P_{B S 1}$, BS1 sends a modified HO_сом message ${ }^{3}$ to the $\mathrm{a} / \mathrm{c}$ in order to start the handover process. When the a/c receives Hо_com with P-bit set ${ }^{4}$, it triggers a LINK_GOING_DOwn message to the network layer, which, in turn, sends a Binding Update (BU) with packet buffering option $^{5}$ (mentioned as "Modified Binding Update (MBU)" in the rest of this paper) to the HA in order to inform not to forward any packets to the currently attached access router and buffer those packets in its queue. In return, the HA sends a Binding Acknowledgment (BA) message with packet buffering option (mentioned as "Modified Binding Acknowledgment (MBA)" in the rest of this paper) to the a/c. After BS1 delivered all packets to the mobile, it sends a HO_com message without the P-bit in order to trigger the layer 2 handover. After the mobile completes layer 2 handover and configures the new $\mathrm{CoA}$, it sends a regular $\mathrm{BU}$ message to the HA, which replies with a BA message and forwards all the buffered packets to the newly attached access router.

\footnotetext{
${ }^{3}$ Details of но_сом message can be found in [12]

${ }^{4} \mathrm{~A}$ new bit, the P-bit, is used from the reserved bit-field in the Hо_cом message. If it is set, the L-DACS 1 BS informs the $\mathrm{a} / \mathrm{c}$ that it has some packets waiting for transmission in its queue. Thus, if the P-bit is not set, no more packets are waiting for the $\mathrm{a} / \mathrm{c}$.

${ }^{5}$ This mobility option is $4 \mathrm{~B}$ long as defined in[21].
} 


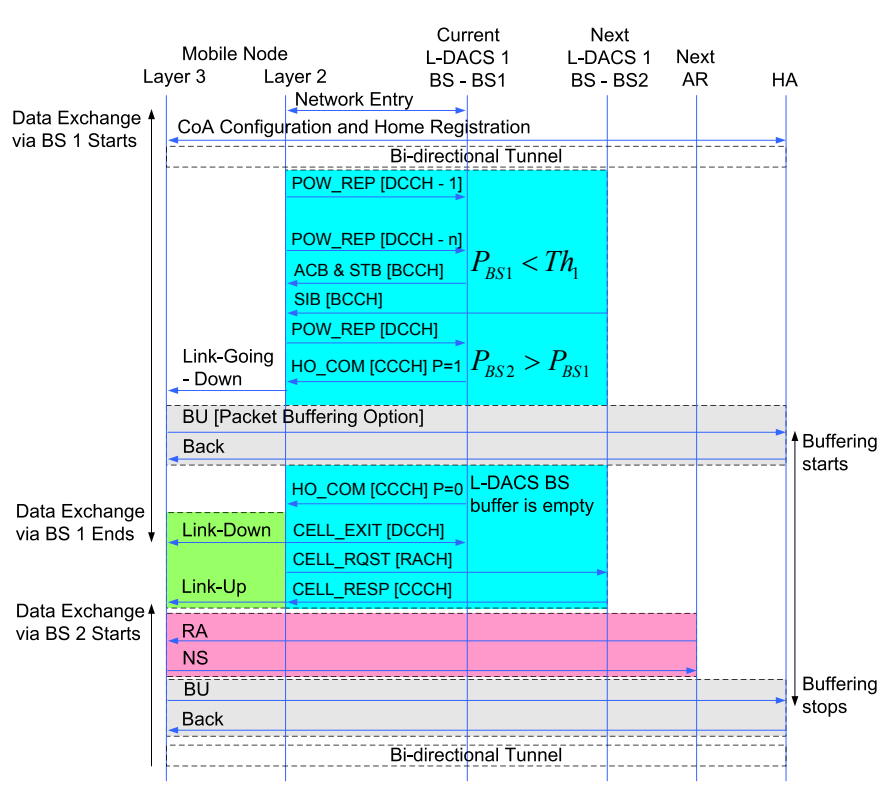

Figure 6: L-DACS 1 handover scenario

\section{Performance Assessment}

We extensively evaluated the proposed handover solution through simulation. We first present our simulation scenario with the considered topology. In order to assess the performance of L-DACS 1 integrated with main network and transport layer functionality, we modeled all the protocols in the OM$\mathrm{NeT}++$ simulation framework[31]. Our main scenario for the performance evaluation is the handover during the download of graphical weather information (WXGRAPH) to an a/c over a TCP connection. The study considered a single user with a dedicated data rate in both base stations. Multiple users and resource allocation strategies are not considered in this paper.

\subsection{Considered Topology}

The main topological considerations for the ATN/IPS are provided in[32]. In our analysis, the European scenario shown in Fig. 7 is considered, where the a/c are communicating with an AOS CN located in Maastricht. An a/c starts communication when it is located in the home domain, performs handover during communication, and moves to the foreign domain. This is a kind of inter-access network handover so that the a/c should complete layer 2 and layer 3 handover procedures in order to continue communication. Within the ground network, the fixed delay values shown in the figure are used. In the aeronautical domain, the cell radii is generally between $150-250 \mathrm{~km}$, and the cell overlapping regions are quite large so that the handover performance does not degrade due to the high speed of an a/c. In our simulations, we consider an a/c with a speed of $800 \mathrm{~km} \mathrm{~h}^{-1}$ with a cell radius of $225 \mathrm{~km}$ and $100 \mathrm{~km}$ distance in the cell overlapping region shown in Fig. $8^{6}$.

\footnotetext{
${ }^{6}$ VHF Datalink Mode 2 (The state-of-the-art technology) is designed to operate with a cell radius of $370 \mathrm{~km}[1]$.
}

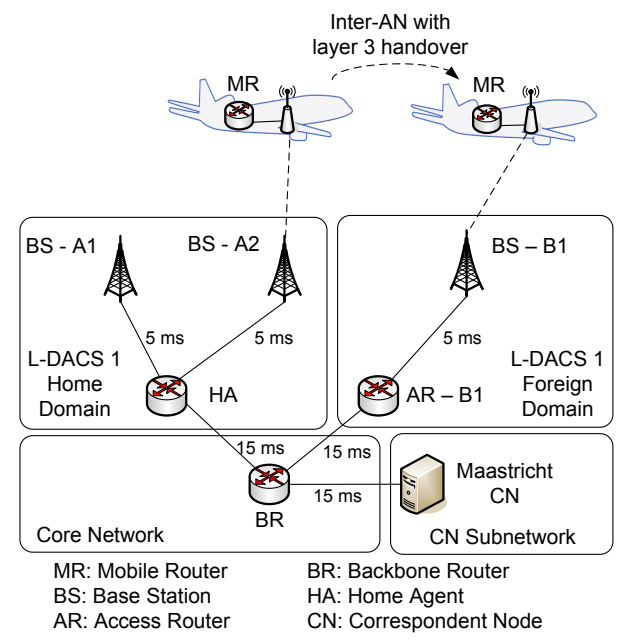

Figure 7: Example topology for the European scenario

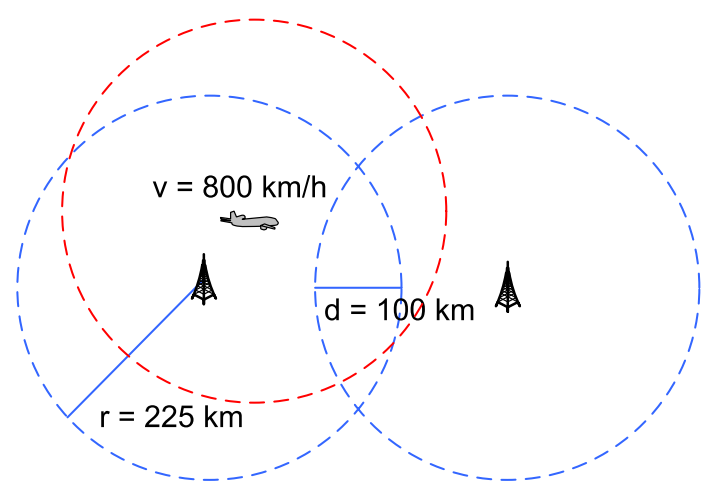

Figure 8: Simulation Configuration

\subsection{Network Layer Considerations}

Table 4 shows the parameters used at the network layer. The first three parameters are related to the DAD and Multicast Listener Discovery (MLD) procedures defined in the neighbor discovery protocol[33]. The last three parameters are specific to the MIPv6 / NEMO protocols and related to home registration[17].

\subsection{Transport and Application Layer Considerations}

In future ATM, three main application types are foreseen, namely, short message exchanges, file transfers, and Voice over IP (VoIP). In this paper, we focus on file transfer. Especially graphical weather information transmission on the forward link

\begin{tabular}{ll}
\hline Parameter & Value \\
\hline IPv6_Default_Dupaddrdetecttrans & 1 \\
IPv6_Default_Retrans_Timer & $1 \mathrm{~s}$ \\
IPv6_Max_Rtr_Solicitation_Delay & $1 \mathrm{~s}$ \\
MIPv6_Initial_Bindack_Timeout & $1 \mathrm{~s}$ \\
MIPv6_Initial_Bindack_Timeout_First & $1.5 \mathrm{~s}$ \\
MIPv6_Max_Bindack_Timeout & $32 \mathrm{~s}$ \\
\hline
\end{tabular}

Table 4: Layer 3 parameters related to the handover 


\begin{tabular}{ll}
\hline Parameter & Value \\
\hline Algorithm & Reno \\
Maximum Segment Size & $1024 \mathrm{~B}$ \\
Receiver Advertised Window & $6 \mathrm{kB}$ \\
Slow Start Threshold & $64 \mathrm{kB}$ \\
\hline
\end{tabular}

Table 5: TCP parameters used in our simulations

\begin{tabular}{llll}
\hline MSS & RTT & $\begin{array}{l}\text { Overhead } \\
\text { home domain }\end{array}$ & $\begin{array}{l}\text { Overhead } \\
\text { foreign domain }\end{array}$ \\
\hline 256 B & $0.27 \mathrm{~s}$ & $20.7 \%$ & $29.5 \%$ \\
$512 \mathrm{~B}$ & $0.295 \mathrm{~s}$ & $11.6 \%$ & $17.3 \%$ \\
$1024 \mathrm{~B}$ & $0.375 \mathrm{~s}$ & $6.1 \%$ & $9.5 \%$ \\
\hline
\end{tabular}

Table 6: RTT and overhead for different MSS values for a $31.5 \mathrm{kbit} \mathrm{s}^{-1}$ link

is considered[8], since it is the main application which generates $80 \%$ of the total data traffic[34, 35], (excluding VoIP traffic). This service requires reliable transmission of $110 \mathrm{kB}$ of information to an a/c. The main TCP standard (RFC 793[36]) is mandated by ATN/IPS[2] for the reliable transport however no additional TCP variant is mandated or proposed. In our simulations, TCP Reno which is implemented by most operating systems [37], is considered as an example transport protocol with the settings shown in Table 5 in order to show the performance gain of HA-buffering method.

\subsection{Bandwidth Delay Product Calculation}

In order to run TCP effectively over a certain wireless link technology, it is important to know the Bandwidth Delay Product (BDP) of the communication path. Table 6 shows the measured round-trip time (RTT) values for different MSS values considering the network topology shown in Fig. 7. In order to measure the RTT values, we consider a simple ping application with the sending rate equal to the L-DACS 1 data rate assigned to the user. The calculated BDP values are around $2 \mathrm{kB}$ and $7 \mathrm{kB}$ for $31.5 \mathrm{kbit} \mathrm{s}^{-1}$ and $158 \mathrm{kbit} \mathrm{s}^{-1}$ data rates, respectively. With those BDP values, L-DACS 1 can be classified as a "Long Thin Network"[38]. The table also shows the protocol overhead for TCP (20 B), IPv6 (40 B), and L-DACS 1 (7 B) for different MSS values. In the case that the $\mathrm{a} / \mathrm{c}$ is in a foreign network, an additional $40 \mathrm{~B}$ is added due to IPv6 tunneling between mobile and its home agent. In our analysis, we consider a MSS value of $1024 \mathrm{~B}$, since it has less overhead and the measured RTT value is only $0.1 \mathrm{~s}$ worse compared to a MSS value of $256 \mathrm{~B}$. This increase is not that critical considering the delay requirement for the WXGRAPH service[8]. As overhead is the more critical issue compared to RTT values in our environment (L-DACS 1 provides limited data rate to users), we consider a MSS of 1024 B in Section 5.6.

Fig. 9 shows the actual RTT values measured when TCP is running with different advertised rwnd values. Looking at Fig. 9(a), $2 \mathrm{kB}, 4 \mathrm{kB}$, and $8 \mathrm{kB}$ rwnd values lead to reasonable RTT values. In the case of $16 \mathrm{kB}$ rwnd, the RTT value reaches up to $4 \mathrm{~s}$, which can be classified as an RTT inflation[39]. RTT

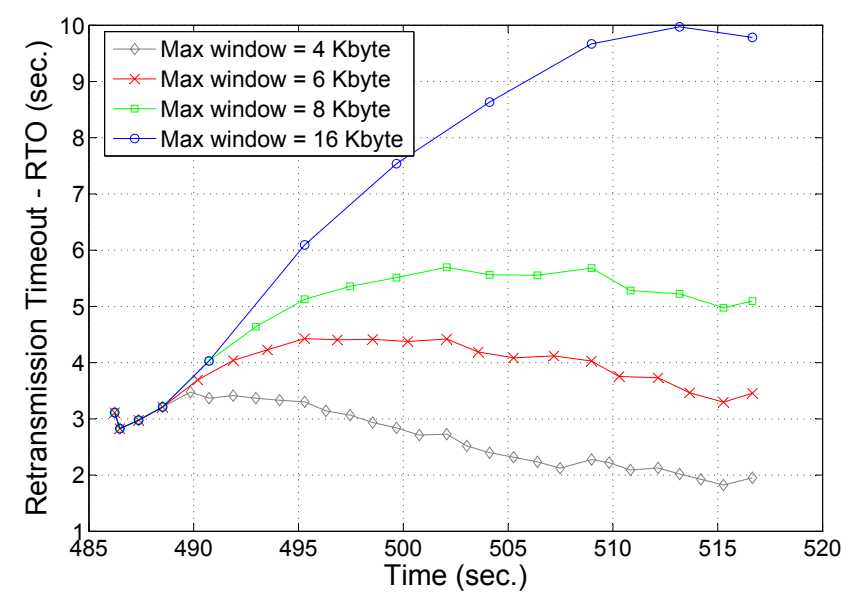

Figure 10: TCP RTO analysis with different maximum window sizes (file transfer of $110 \mathrm{~KB}$, data rate per user of $31.5 \mathrm{kbit} \mathrm{s}^{-1}$, MSS of 1024

inflation is mainly due to L-DACS 1's excessive queuing delay. This is also causing retransmission timeout (RTO) inflation as shown in Fig. 10, so that the TCP sender becomes less reactive due to the higher recovery time, which, in turn, causes worse TCP recovery in case a handover events. Here, RTO is calculated via smoothed round-trip time (SRTT) and round-trip time variation (RTTVAR) parameters, which are calculated based on Jacobson's algorithm[40]. On the other side, when rwnd is set to $2 \mathrm{kB}$ and the user data rate is higher $\left(158 \mathrm{kbit} \mathrm{s}^{-1}\right)$, the link is under utilized so that the transmission is completed around $7 \mathrm{~s}$ later compared to the $8 \mathrm{kB}$ rwnd as shown in Fig. 9(b). In this case, $8 \mathrm{kB}$ and $16 \mathrm{kB}$ rwnd provide reasonable RTT values.

Please also note that using such low advertised window sizes $(6 \mathrm{kB})$ is different than normal practice where either buffer autotuning is used (where the receiver gradually opens up the advertised receive window) or where large advertised windows are used, and the congestion window management and loss recovery algorithm determines performance. In our case, we should pay attention not to set the advertised window size too low since it creates an upper-bound on the transmission rate and may cause under utilization of the link as shown in $2 \mathrm{kB}$ case of Fig. 9(b). Therefore, in order to set the reasonable advertised window size, mobile user should have the knowledge of the BDP of the link. One approach is to consider cross layer information such that link technology measures the RTT and instantaneous throughput and sends this information to TCP in order to adjust the advertised window size[41, 42]. Another approach is to set receiver window size dynamically by measuring the bandwidth and the RTT of the TCP connection as proposed by $[43]^{7}$. In the following section, we consider a $31.5 \mathrm{kbit} \mathrm{s}^{-1}$ user data rate and a $6 \mathrm{kB}$ of advertised rwnd for our analysis.

\subsection{Further Assumptions}

We further assume that the buffer size of BS is large enough so that no packet drops occur at the BS due to buffer overflow. In addition, wireless channel loss (i.e., the FER) is modeled as

\footnotetext{
${ }^{7}$ Different proposals related to TCP buffer auto-tuning can be found in [44].
} 


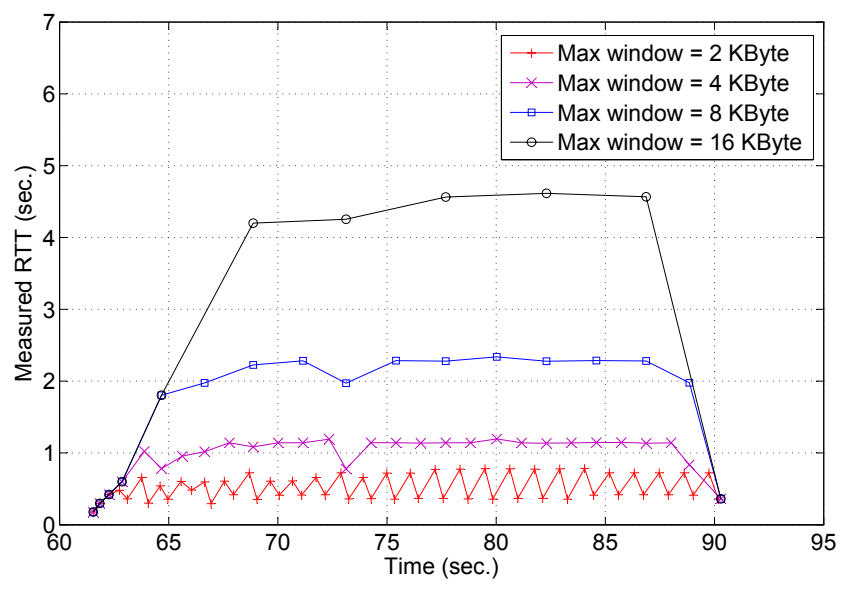

(a) RTT with different advertised window sizes, data rate per user of $31.5 \mathrm{kbit} \mathrm{s}^{-1}$, MSS of 1024

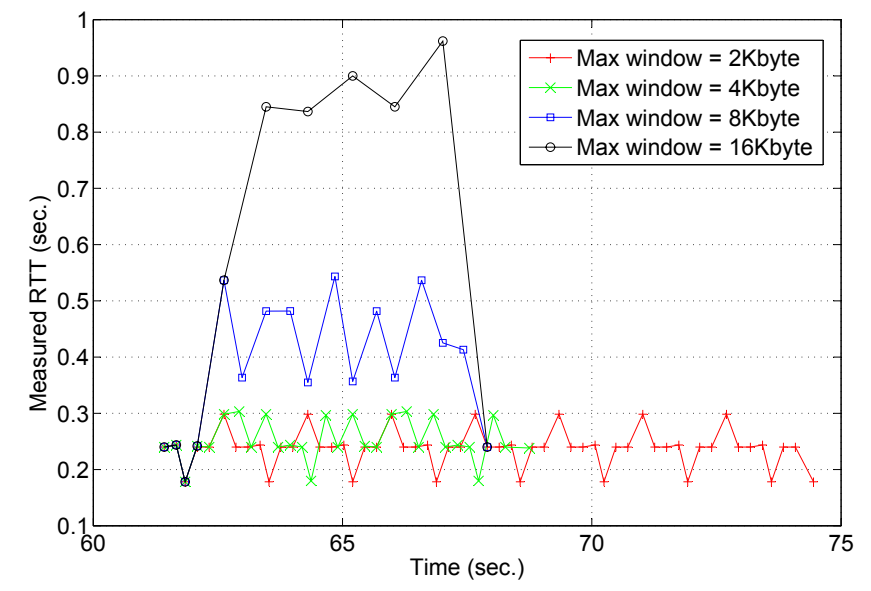

(b) RTT with different advertised window sizes, data rate per user of $158 \mathrm{kbits}^{-1}$, MSS of 1024

Figure 9: RTT analysis with different advertised window sizes and L-DACS 1 data rates during $110 \mathrm{~KB}$ file transfer

i.i.d. with the values shown in Section 2.4. The standard TCP ACK scheme is considered, i.e. no delayed TCP ACKs.

We also assume that the allocated user data rate in both cells is the same (i.e., $31.5 \mathrm{kbit} \mathrm{s}^{-1}$ ) and that all L-DACS 1 specific messages are transmitted without any channel error. ARQ is enabled with at most five retransmission opportunities for the user data.

\subsection{Simulation Results and Analysis}

We define three different scenarios, namely, baseline, proposal 1 , and proposal 2. The baseline scenario refers to[10], which is mainly using a link layer trigger (IEEE 802.21 functionality) in the BS to send a Router Advertisement (RA) message to the mobile after the layer 2 handover is completed. Proposal 1 refers to the addition of the DAD modifications mentioned in Section 3.2. In addition, proposal 2 relies on the addition of HA-buffering method mentioned in Section 4.

Fig. 11 shows the TCP transmission completion duration of $110 \mathrm{kB}$ of file transfer for the three scenarios. These values are taken from 20 simulation runs where each run simulates one $\mathrm{a} / \mathrm{c}$ handover instance. Both the baseline and proposal 1 show similar performance as both approaches wait for the TCP sender timeout (i.e., the RTO timer expiry) so that the new packets will be sent to the new link. In a few cases, the TCP sender goes to two timeouts in baseline, because the handover is completed after the first timeout occurrence at the TCP sender. In this case, the transmission completion time increases for about $8 \mathrm{~s}$ since the retransmission timer value increases exponentially in every timeout. In proposal 2, because HA forwards the buffered traffic after the handover is completed, the TCP receiver continues to send ACKs to the TCP sender, which, in turn, causes transmission of new packets by the TCP sender. In this case, the transmission is completed almost $4 \mathrm{~s}$ earlier on average compared to proposal 1 .

Fig. 12 plots the received TCP sequence numbers over time for all three scenarios considering a single simulation run. In baseline and proposal 1 , the handover starts at around $\mathrm{t} 1$ (at

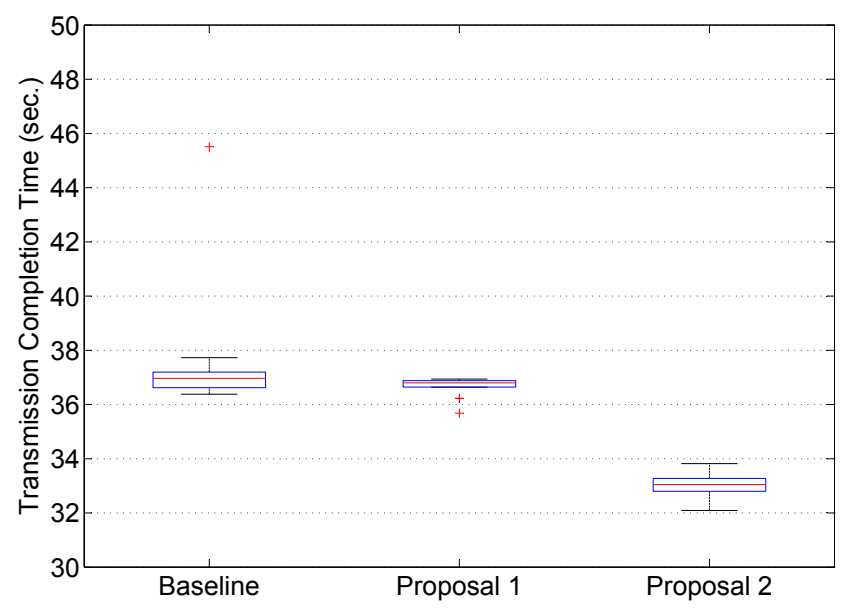

Figure 11: TCP transmission completion time

$500.2 \mathrm{~s}$ ). However, in proposal 2, it starts about $1.9 \mathrm{~s}$ later (t4). This is due to the fact that in proposal 2, the currently attached BS continues to deliver the packets in its buffer before it initiates the handover process. In the figure, $\mathrm{t} 2, \mathrm{t} 3$, and $\mathrm{t} 5$ show the handover completion time of proposal 1, baseline and proposal 2 , respectively. It is also good to mention that due to the ARQ component, all TCP packets are delivered to a/c without any loss due to wireless channel effects in normal situation (i.e., from TCP session established to TCP session closed except handover duration).

\subsection{Relation Between Handover Completion Time and TCP RTO Expiry Time}

In case that the handover completion time is larger than the TCP RTO expiry time, the HA will also buffer all the retransmitted packets. If such a case occurs, the HA should drop the TCP packets with the same sequence number in order not to overload the L-DACS 1 BS by sending multiple copies of the same packet. However, if those packets are IPSec protected, it is 


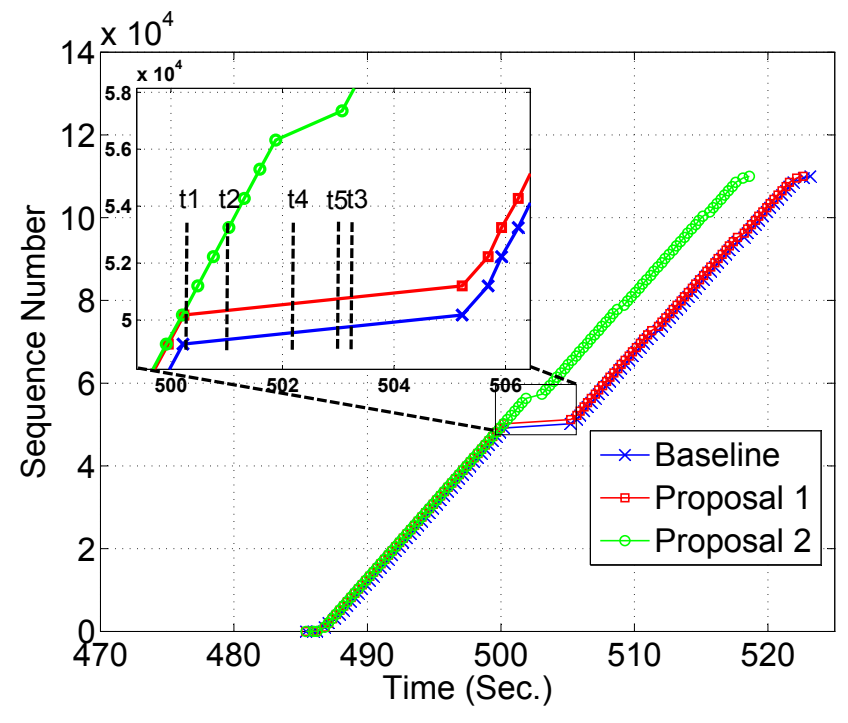

Figure 12: Received TCP sequence number

not possible to check the sequence numbers - other solutions are required. In our case, the situation is completely different since handover completion time in proposal 2 (about $0.8 \mathrm{~s}$ ) is significantly shorter than the TCP RTO expiry time (about $4 \mathrm{~s}$ when advertised rwnd is $6 \mathrm{kB}$, cf. Fig. 10), so that HA only buffers the packets that are sent by TCP sender for the first time.

\section{Mobility Signaling Message Loss Conditions in HA Buffering Method}

In this section, we discuss possible scenarios when a mobility signaling message is lost due to mainly wireless channel errors. Normally, the loss probability of a MBU in the RL and MBA message in the FL are 0.322 and $6.31 \times 10^{-5}$, respectively. However, due to the ARQ component of L-DACS 1, the loss probabilities get reduced to about $1.1 \times 10^{-3}$ for the MBU in the RL and to $6.25 \times 10^{-26}$ for the MBA in the FL with five retransmission possibilities. Here we assumed that the each retransmission is an independent event.

After the first specification of L-DACS 1 was released, the high Packet Error Rate (PER) values in the RL were noticed when the mobile sends a message with multiple OFDM frames in a multiframe. This is due to the fact that each L-DACS 1 frame (14 B length with lowest modulation and coding) is coded and interleaved separately. When a higher layer message is sent with multiple frames, then the loss probability of the higher layer message becomes quite high. Recently, an update of the L-DACS 1 specification has been developed within the Single European Sky ATM Research (SESAR) project P15.2.4[45], which deals among others with the L-DACS development. In the updated specification, coding and interleaving is performed jointly for all frames transmitted by an a/c which results in significantly reduced PER. In the considered case, the loss probability of MBU message becomes $1 \times 10^{-2}$ instead of 0.322 for a single transmission.

\subsection{Impact of Signalling Message Losses on the Handover Performance}

We have identified four important conditions related to mobility signaling message losses, which we discuss in the following. The first two conditions are related to loss of MBU messages and loss of MBA messages, respectively followed by successful regular home registration procedure. The other two conditions are specifically tied to the loss of MBA message followed by delayed regular home registration procedures which is due to either loss of regular BU or loss of regular BA message.

First condition - loss of all MBU messages before handover with regular home registration after handover:. When the mobile sends a MBU, it starts a timer which is initially set to $1 \mathrm{~s}$. In case it does not receive a MBA within $1 \mathrm{~s}$, it retransmits another MBU. After three retransmission, it stops the retransmission and waits for the Hо_com message (with P-bit not set) to perform regular handover. NB: If the MBA message is not received by the mobile, it is not aware whether the MBU or the MBA was dropped.

In the case that all MBU messages are dropped, i.e. not received by the HA, the HA will continue the normal forwarding operation for incoming packets to the currently attached L-DACS 1 BS. Meanwhile BS informs the mobile that it still has some packets by sending a но_сом (with P-bit set). In parallel, the BS continues to check the received power level and, when the received power drops below a certain threshold $T h_{2}$, it initiates the handover by sending a Hо_com message (with P-bit not set). So that mobile starts layer 2 handover followed by regular home registration procedure. This condition is more like a forced handover. In such a case, the performance of the HA-buffering (proposal 2) will be the same as with proposal 1. The only difference is that the handover will start later (in the order of a few seconds) compared to proposal 1.

Second condition - loss of all MBA messages before handover with regular home registration after handover:. If a MBA message is lost, the mobile sends another MBU after the timer expires, which, in turn, triggers the transmission of another MBA. In the case that all MBA messages are not received by the mobile, it does not affect the performance of the protocol since the HA already received the MBU and starts buffering the traffic. Similar to the first condition, the mobile has no information whether the MBU or the MBA got lost.

The HA stops forwarding the traffic to the current L-DACS 1 BS when it receives MBU message. Afterwards, when the BS queue becomes empty, BS will send a HO_com (with P-bit not set) to the mobile, which starts layer 2 handover followed by regular home registration procedure. So, there is no negative outcome of this condition and the a/c still uses the HA-buffering functionality.

Third condition - loss of regular BU message after handover with loss of all MBA messages before handover:. In this case, HA continues to buffer the traffic until it receives the regular BU message. If regular BU reception is delayed due to losses, then the handover completion time and in parallel the reception of 
the first packet over the new link is also delayed. In case, the TCP sender receives the first ACK from the new link before TCP RTO timer expiry, then the TCP sender will not go to timeout and will continue its regular operation. In other case (i.e., the reception of first ACK after TCP RTO expiry) the TCP sender will go to timeout and will retransmit unacknowledged packets. In this case, HA should do the operation defined in Section 5.7.

In case the HA receives the regular BU after TCP RTO timer expiry, it starts to receive copies of messages it already received and acts as described in Section 5.7. We will again observe the same TCP retransmission behavior in baseline and proposal 1 scenario due to TCP RTO timer expiry. However in the HAbuffering case, if the HA does not drop the packets with the same sequence number, it will cause additional traffic on the newly attached L-DACS 1 BS.

Fourth condition - loss of first regular BA message after handover with loss of all MBA messages before handover:. In this case, the HA receives regular BU message and creates an entry in the binding cache and forwards the buffered packets to the mobile. However, since the mobile cannot receive the first regular BA message, it does not create an entry in its binding update list. Thus, the mobile drops the forwarded packets and waits until MIPv6_INITIAL_BINDACK_TIMEOUT timer expiry in order to retransmit a new $\mathrm{BU}$.

As the HA forwards the buffered packets to the L-DACS 1 $\mathrm{BS}$, those packets consume a certain amount of resources on the FL although they will be dropped by the a/c due to the loss of regular BA message.

In addition, loss of regular BA messages increases the handover completion time in all three scenarios.

\section{HA Buffering Load Considerations}

As mentioned in Section 1, when an a/c is flying in TMA/En Route service volumes, it normally communicates with two CNs. One $\mathrm{CN}$ is for the air traffic services and one for the airline operations. WXGRAPH information is received from AOS CN and normally represent $80 \%$ of the total traffic. Another message type is common trajectory coordination (COTRAC), which requires transmission of about $6 \mathrm{kB}$ in the forward link and $5 \mathrm{kB}$ in the return link. The remaining network traffic consists of small message exchanges of a few $100 \mathrm{~B}$ per message. This means, mostly WXGRAPH traffic will be affected by the handover events due to higher load and longer transmission time. Therefore, we can say the number of flows running in parallel in aeronautical domain is quite limited per a/c, which, in turn, can be handled using the HA-buffering method. However, assuming the use of consumer electronics, multiple long-lasting flows can be expected. Another important criteria is the number of mobile users (i.e., a/c), which is in the order of a several thousand in the aeronautical environment[1]. This can easily be handled by HA-buffering. Again, assuming the presence of consumer electronics, this number could be in the order of a few million. In future work, the scalability of the HA-buffering needs to be evaluated under these assumptions.

\section{Conclusion}

In this paper, we further extended our previous study[10] in two ways. First, we provided two solutions in order to remove the DAD procedure in order to further decrease the handover delay. In the second part, we integrated the HA-buffering method into our network. We have shown clear performance improvements in TCP during the handover for download of a typical file sizes of $110 \mathrm{kB}$. We realized that proposal 1 does not improve the TCP performance compared to the baseline in most cases. However, we noticed that the TCP sender sometimes experiences two timeouts in the baseline scenario which degrades its performance significantly compared to proposal 1. From an application's perspective, VoIP traffic will definitely benefit from proposal 1 improvements since the handover delay is reduced to around $0.8 \mathrm{~s}$. With proposal 2 , we kept the handover delay at the same level as for proposal 1. However, thanks to the HAbuffering method, the TCP receiver does not has to wait for the TCP sender timeout in order to receive the packets after the handover completed. The resulting TCP completion time is reduced by about $10 \%$ for a transmission of $110 \mathrm{kB}$ over $31.5 \mathrm{kbit} \mathrm{s}^{-1}$ wireless link.

As a future work, we will study the performance of HAbuffering on TCP in case the a/c performs handover between BSs which provide different data rates to the a/c (i.e. handover from low bandwidth to high bandwidth data link or vice versa). In this case, it is important to set the TCP window size dynamically as proposed by[41, 42]. In addition, we are also planning to investigate the performance gain of HA-buffering with different TCP variants like Selective Acknowledgment (SACK) option[46] and NewReno[47].

\section{References}

[1] W. M. Eddy, W. Ivancic, T. Davis, NEMO Route Optimization Requirements for Operational Use in Aeronautics and Space Exploration Mobile Networks, RFC 5522, IETF (October 2009).

[2] Manual for the ATN using IPS Standards and Protocols, Tech. Rep. Doc 9896-AN/469, ICAO (2009).

[3] V. Devarapalli, R. Wakikawa, A. Petrescu, P. Thubert, Network Mobility (NEMO) Basic Support Protocol, RFC 3963, IETF (January 2005).

[4] J. Lai, Y. A. Sekercioglu, N. Jordan, A. Pitsillides, Performance Evaluation of Mobile IPv6 Handover Extensions in an IEEE 802.11b Wireless Network Environment, in: 11th IEEE Symposium on Computers and Communications (ISCC 2006), IEEE, Sardinia, Italy, 2006, pp. 161-166.

[5] G. Xie, J. Chen, H. Zheng, J. Yang, Y. Zhang, Handover Latency of MIPv6 Implementation in Linux, in: IEEE Global Telecommunications Conference (IEEE GLOBECOM 2007), IEEE, Washington, DC, 2007, pp. 1780-1785.

[6] J. Pinola, K. Pentikousis, IPTV over WiMAX with MIPv6 Handovers, in: 69th IEEE Vehicular Technology Conference (VTC2009-Spring), IEEE, Barcelona, Spain, 2009, pp. 1-5.

[7] H. Petander, E. Perera, K.-C. L. A. Seneviratne, Measuring and Improving the Performance of Network Mobility Management in IPv6 Networks, IEEE Journal on Selected Areas in Communications (JSAC) 24 (9) (2006) 1671-1681.

[8] Communications Operating Concepts and Requirements for the Future Radio System, Tech. rep., Eurocontrol/FAA (May 2007).

[9] C. Bauer, S. Ayaz, A Thorough Investigation of Mobile IPv6 for the Aeronautical Environment, in: 68th IEEE Vehicular Technology Conference (VTC2008-Fall), IEEE, Calgary, BC, Canada, 2008, pp. 1-5.

[10] S. Ayaz, F. Hoffmann, C. Sommer, R. German, F. Dressler, Performance Evaluation of Network Mobility Handover over Future Aeronautical Data 
Link, in: IEEE Global Telecommunications Conference (GLOBECOM 2010), IEEE, Miami, FL, 2010.

[11] S. Brandes, U. Epple, S. Gligorevic, M. Schnell, B. Haindl, M. Sajatovic, Physical layer specification of the L-band Digital Aeronautical Communications System (L-DACS1), in: Integrated Communications, Navigation and Surveillance Conference (ICNS'09), Arlington, VA, 2009, pp. 1-12.

[12] L-DACS1 System Definition Proposal: Deliverable D2, Tech. rep., Eurocontrol (February 2009).

[13] L-DACS2 System Definition Proposal: Deliverable D2, Tech. rep., Eurocontrol (May 2009).

[14] C. So-In, R. Jain, A.-K. Tamimi, Scheduling in IEEE 802.16e mobile WiMAX networks: key issues and a survey, IEEE Journal on Selected Areas in Communications (JSAC) 27 (2) (2009) 156-171. doi:10.1109/JSAC.2009.090207.

[15] E. Haas, Aeronautical channel modeling, IEEE Transactions on Vehicular Technology 51 (2) (2002) 254-264.

[16] S. Brandes, U. Epple, M. Schnell, Compensation of the Impact of Interference Mitigation by Pulse Blanking in OFDM Systems, in: IEEE Global Telecommunications Conference (GLOBECOM 2009), Honolulu, HI, 2009, pp. 1-6. doi:10.1109/GLOCOM.2009.5426056.

[17] D. B. Johnson, C. E. Perkins, J. Arkko, Mobility Support in IPv6, RFC 3775, IETF (June 2004).

[18] R. M. Hinden, S. E. Deering, IP Version 6 Addressing Architecture, RFC 4291, IETF (February 2006).

[19] Non-Access-Stratum (NAS) protocol for Evolved Packet System (EPS), Technical Specification TS 24.301 V10.0.0, 3GPP (October 2010).

[20] Access to the 3GPP Evolved Packet Core (EPC) via non-3GPP access networks, Technical Specification TS 24.302 V10.2.0, 3GPP (December 2010).

[21] F. Xia, B. Sarikaya, B. Patil, Mobile IPv6 Handover Using Home Agent Buffering, I-D draft-xia-mipshop-ha-buffering-01, IETF (November 2008).

[22] P. S. Henry, H. Luo, Buffering packets destined for a mobile device while the mobile device moves from one network to another network to prevent handoff packet loss, United States Patent 7600040, AT\&T Corporation (October 2009)

[23] H.-D. Park, Y.-H. Kwon, K.-W. Lee, Y.-S. Choi, S.-H. Lee, Y. Z. Cho, Network Mobility Management Using Predictive Binding Update, in: 7th International Workshop on Distributed Computing (IWDC 2005), Vol. LNCS 3741, Springer, Kharagpur, India, 2005

[24] K. Hur, K. H. Tchah, D. S. Eom, TCP Performance Analysis of Packet Buffering in Mobile IP Based Networks, IEICE Transactions on Communications E87-B (11) (2004) 3361-3369.

[25] S. B. Lee, K. Hur, J. Park, D.-S. Eom, A packet forwarding controller for mobile IP-based networks with packet buffering, IEEE Transactions on Consumer Electronics 55 (3) (2009) 1344-1350.

[26] Y.-S. Kim, D.-H. Kwon, Y.-J. Suh, Seamless handover support over heterogeneous networks using FMIPv6 with definitive L2 triggers, Wireless Personal Communications 43 (2007) 919-932.

[27] D. S. Eom, M. Sugano, M. Murata, H. Miyahara, Performance Improvement by Packet Buffering in Mobile IP Based Networks, IEICE Transactions on Communications E83-B (11) (2000) 2501-2512.

[28] D. Le, X. Fu, D. Hogrefe, A Cross-Layer Approach for Improving TCP
Performance in Mobile Environments, Wireless Personal Communications 52 (2010) 669-692.

[29] L. Daniel, M. Kojo, Employing cross-layer assisted TCP algorithms to improve TCP performance with vertical handoffs, International Journal of Communication Networks and Distributed Systems 1 (2008) 433-465.

[30] D. Lee, J. Kim, TCP Performance Enhancement Incorporating Handoff Analysis in Mobile IPv6 Networks, in: High Speed Networks and Multimedia Communications, Vol. 3079 of LNCS, Springer, 2004, pp. 512-523.

[31] A. Varga, The OMNeT++ Discrete Event Simulation System, in: European Simulation Multiconference (ESM 2001), Prague, Czech Republic, 2001.

[32] C. Bauer, S. Ayaz, ATN Topology Considerations for Aeronautical NEMO $\mathrm{RO}$, Internet-Draft (work in progress) draft-bauer-mext-aero-topology01.txt, IETF (September 2009).

[33] T. Narten, E. Nordmark, W. A. Simpson, H. Soliman, Neighbor Discovery for IP version 6 (IPv6), RFC 4861, IETF (September 2007).

[34] E. Allaix, T. Azzarelli, Estimation of Information Volume Requirements for ATM under AI 1.7, Working Paper WP17, ICAO (April 2009).

[35] M. Ehammer, T. Graupl, C.-H. Rokitansky, TCP/IP over aeronautical communication systems - Effects on bandwidth consumption, in: 28th IEEE/AIAA Digital Avionics Systems Conference (DASC'09), Orlando, FL, 2009, pp. 4.A.3-1 -4.A.3-9.

[36] J. Postel, Transmission Control Protocol, RFC 793, IETF (September 1981).

[37] V. Gupta, S. Dharmaraja, M. Gong, Analytical modeling of TCP flow in wireless LANs, Mathematical and Computer Modelling 53 (5-6) (2011) 684-693. doi:http://dx.doi.org/10.1016/j.mcm.2010.10.004.

[38] G. E. Montenegro, S. Dawkins, M. Kojo, V. Magret, N. Vaidya, Long Thin Networks, RFC 2757, IETF (January 2000).

[39] R. Chakravorty, J. Cartwright, I. Pratt, Practical experience with TCP over GPRS, in: IEEE Global Telecommunications Conference (GLOBECOM 2002), Vol. 2, Taipei, Taiwan, 2002, pp. 1678-1682.

[40] V. Jacobson, Congestion avoidance and control, ACM SIGCOMM Computer Communication Review 18 (1988) 314-329.

[41] Y. Gou, D. Pearce, P. Mitchell, A receiver-based vertical handover mechanism for TCP congestion control, IEEE Transactions on Wireless Communications 5 (10) (2006) 2824-2833.

[42] N. Kirschnick, F. Steuer, P. Vidales, S. Albayrak, Adaptive window size to reduce the influence of heterogeneous mobility on TCP performance, in: IEEE Symposium on Computers and Communications (ISCC 2008), Marrakech, Morocco, 2008, pp. 149-156.

[43] M. E. Fisk, W. Feng, Dynamic Right-Sizing in TCP, in: 2nd Annual Los Alamos Computer Science Institute Symposium, Santa Fe, MX, 2001

[44] E. Weigle, W.-c. Feng, A Comparison of TCP Automatic Tuning Techniques for Distributed Computing, in: 11th IEEE International Symposium on High Performance Distributed Computing (HPDC 2002), IEEE, Washington, DC, 2002, p. 265.

[45] WP15 - Non Avionic CNS System, Description of work, SESAR Joint Undertaking (December 2008).

[46] M. Mathis, J. Mahdavi, S. Floyd, A. Romanow, TCP Selective Acknowledgment Options, RFC 2018, IETF (October 1996).

[47] S. Floyd, T. Henderson, A. Gurtov, The NewReno Modification to TCP's Fast Recovery Algorithm, RFC 3782, IETF (April 2004). 